\title{
The clinical features of posterior scleritis with serous retinal detachment: A retrospective clinical analysis
}

\section{Zhizhang Dong}

The second affiliated hospital and Yuying Children's hospital of Wenzhou Medical University https://orcid.org/0000-0001-5150-8750

\section{Yifeng Gan}

The second affiliated hospital and Yuying Children's hospital of Wenzhou Medical University

\section{Yinan Zhang}

The second affiliated hospital and Yuying Children's hospital of Wenzhou Medical University

\section{Yu Zhang}

The second affiliated hospital and Yuying Children's hospital of Wenzhou Medical University

Juan Li ( $\sim$ cornea@163.com )

Guangren Hospital Affiliated to School of Medicine of Xi'an Jiaotong University

\section{Haihua Zheng ( $\nabla$ eyezhh@126.com )}

The second affiliated hospital and Yuying Children's hospital of Wenzhou Medical University

\section{Research Article}

\section{Keywords:}

Posted Date: November 30th, 2018

DOI: https://doi.org/10.21203/rs.2.48/v1

License: (c) (1) This work is licensed under a Creative Commons Attribution 4.0 International License. Read Full License

Version of Record: A version of this preprint was published at International Journal of Ophthalmology on July 18th, 2019. See the published version at https://doi.org/10.18240/ijo.2019.07.16. 


\section{Abstract}

Objective: To summarize the clinical features, systemic associations, risk factors and choroidal thickness(CT) changing in posterior scleritis (PS) with serous retinal detachment.

Methods: This retrospective study included 23 patients with PS with retinal detachment from August 2012 to July 2017. All patients were documented with the Medical history and clinical features were recorded. The examinations included best corrected visual acuity (BACV), intraocular pressure区IOP区, fundus examination, routine eye examinations. Posterior coats thickness (PCT) was determined by B scan Ultrasound, the CT was measured by enhanced depth imaging spectral-domain optical coherence tomography (EDI-OCT). And clinical data were compiled and analyzed.

Results: After application of extensive exclusion criteria, 23 patients with PS remained (13 females, 10 males). The average age at presentation was $29.5 \pm 9.24$ years old. Ocular pain and blurred vision were the two most common symptoms complained by patients. Anterior scleritis occurred in 12 patients, which was confirmed by Ultrasound Biomicroscopy (UBM) examination. Despite all patients displaying serous retinal detachment in their macula, no fluorescein leakage was observed in the macular. Optic disc swelling was documented in 10 of the 23 eyes. From B-scan ultrasound examination, the PCT increased with fluid in Tenon's capsule demonstrated as a typical T-sign. The average PCT was $2.51 \pm 0.85 \mathrm{~mm}$ in the PS-affected eye and only $1.09 \pm 0.29 \mathrm{~mm}$ in the unaffected eye; this difference reached statistical significance. The subfoveal CT increased to an average of $442.61 \pm 55.61 \mu \mathrm{m}$, which correlated with axis length and PCT, but not with IOP. The BCVA and IOP did not correlate with either CT or the PCT.

Conclusions: PS with serous retinal detachment presented with a variety of symptoms, such as pain and visual loss, and physical indicators. Typical T-sign detected by B-scan ultrasound was a useful confirmatory sign for PS diagnosis. Pathological increases in CT might be a potential predictive factor for inflammation.

\section{Introduction}

Posterior scleritis (PS) is an uncommon and under-diagnosed condition caused by inflammation of the sclera (i.e. the fibrous outer layer of the eye) [1]. Due to its low incidence and variable clinical presentation, the mechanism underlying PS remains unclear and the majority of the research to date has focused instead on characterizing its clinical features, and optimizing diagnosis, treatment, and patient outcome[2-4]. Based on the anatomical location, PS presents with a range of clinical features, especially in fundus change, and, therefore, is often misdiagnosed as intraocular inflammation[5], ocular tumors[6], or orbital inflammation[7]; such misdiagnoses[8] increase the likelihood of incurring irreversible visual damage including eventual vision loss. Given the high rates of retinal detachment in PS[4], this condition can be easily misdiagnosed as central serous chorioretinopathy (CSC), which is characterized by retinal detachment in or around the macula. There is currently a paucity of research dedicated to detailing the differences between these two diseases. These factors motivated our interest in the clinical features and risk factors involved in PS with 
retinal detachment specifically. This research was performed toward fully characterizing the clinical features, systemic associations, and risk factors in PS with retinal detachment.

Recently some studies have suggested that choroidal expansion might play an important role in PS[9, 10]. Due to advances in ophthalmic imaging equipment, choroidal thickness (CT) can be noninvasively measured by enhanced depth imaging optical coherence tomography (EDI-OCT). While studies have implicated the importance of CT in PS, but none have focused on how CT is involved in PS with retinal detachment $[10,11]$. Furthermore, CT is increasingly being considered as an important risk factor in the pathophysiology of PS. However, little is known about the association between CT and other ocular biometric parameters, such as visual acuity, axial length (AL) and posterior coats thickness (PCT), in PS, let alone in PS with retinal detachment. Therefore, this study aimed to investigate the clinic findings and association of CT in PS with serous retinal detachment by a retrospective clinical analysis.

\section{Methods}

\section{Subjects and Enrollment Criteria}

This study was approved by the Ethical Review Committee of the Second Affiliated Hospital of Wenzhou Medical University, Wenzhou City, China. All procedures adhered to the tenets of the Declaration of Helsinki for research involving human subjects. Written informed consent was obtained from all participants enrolled in this study. All potential study participants were from a Chinese Han population and patients of the aforementioned clinic.

A retrospective review of patient medical records from the ophthalmological clinic at the Second Affiliated Hospital of Wenzhou Medical University between 2012 and 2017 was performed to identify patients with a diagnosis of PS.

For the purpose of this study, PS was diagnosed by presence of (1) acute or sub-acute symptom onset; (2) eye pain with or without decreased visual acuity, accompanied by various fundus changes (e.g. optic disc edema, retinal phlebectasia, exudative retinal detachment); (4) eyeball thickening and fluid in the Tenon's capsular with low echo and fascial sac edema in the eye coats, defined as typical "T" sign[3, 4, 12], revealed by B-scan ultrasound examination;

Patients with any of the following conditions were excluded: incomplete clinical information, infection with acute orbital cellulitis, tuberculosis, or syphilis; trauma; tumor; the orbital inflammatory pseudotumor; Grave's ophthalmopathy; macular retinal detachment with abnormal leakage in fundus fluorescein angiography; rhegmatogenous retinal detachment; any obvious cataract leading to an intumescent lens; a history of intraocular surgery; inability to tolerate ophthalmological examination; clinically relevant opacities of the optic media; and low-quality images due to unstable fixation or a severe cataract.

Of the 69 records initially identified, 16 were excluded because of insufficient clinical detail to ensure a correct diagnosis or lack of satisfactory ultrasonography data. 30 PS patients were not included because no sub retinal fluid was detected in our research. 
After the application of the inclusion and exclusion criteria, 23 remaining patients were included in this study. All enrolled patients were diagnosed with PS presenting serous retinal detachment over the macula.

\section{Study Measurements}

Demographic data on age, sex, and blood pressure were collected. Detailed clinical information regarding original diagnosis, disease onset, clinical presentation, ultrasound data, and systemic associations was recorded. All patients were regularly tested with regular blood test, CRP, ERS, P-ANCA, C-ANCA, ANA, PANA, "ASO" antigen test, dsDNA antibody, SS-A and SS-B antibody, complement series, SAA, RF factor, Immunoglobulin $\mathrm{G}$ and $\mathrm{M}$. All subjects underwent a thorough ophthalmic evaluation, including the best corrected visual acuity (BCVA) assessment, slit-lamp biomicroscopy, intraocular pressure (IOP) measurement (applanation tonometry), fundus examination, ultrasonographic biomicroscopy, ultrasound (UBM), B-mode scanning (B-scan, Bio-vision Echosens, France), fundus fluorescein angiography (FFA), refractive error examination, and Axial Length $(A L)$ measured with partial optical coherence interferometry (IOL Master; Carl Zeiss Meditec, German). All examinations were performed upon diagnosis by experienced ophthalmologists who are blinded to the patients diagnosis.

\section{EDI-OCT Examination}

As previously described[13], CT was determined using the Spectralis device (Heidelberg Engineering, Heidelberg, Germany), by using the device's automatic averaging (about 100 real-time frames were averaged) and eye-tracking features. First of all, in order to estimate optical magnification and achieve accurate comparisons across individuals, keratometry readings and the refraction data were collected and entered into the Spectralis software program. For the aim of ensuring high-quality images, Tthe quality of choroidal imaging was judged according to the signal-to-noise ratio, and only images ratios $\geq 20 \mathrm{~dB}$ were collected and used for further analysis. The resulting scans were visualized and measured by the measuring software, the standard Spectralis OCT (v1.5.12.0; Heidelberg Engineering). The choroid thickness was measured from the outer portion of the retinal pigment epithelium to the inner surface of the sclera as indicated in the Fig. $1 \mathrm{C}$ by arrow. CT was measured at the fovea in all subjects. wo experienced ophthalmologists, blinded to the clinical diagnoses of the patients, performed EDI-OCT examinations and measurements and the latter were averaged before further analysis.

\section{Statistical Analysis}

Data were analyzed using SPSS 17.0 software (SPSS, Chicago, IL, USA). Before analysis, we used the SPSS software to confirm that data from all variables were normally distributed. A paired t-test was used to determine changes in macular CT. Pearson's correlation analysis was performed to evaluate the relationships between the changes in CT and BCVA, AL, and PCT. For these tests, a p-value of $<0.05$ was considered statistically significant.

\section{Results}


During the five-year period from August 2012 to July 2017, nearly 300,000 patients were referred to the Ophthalmology Department of the Second Affiliated Hospital and Yuying Children's Hospital of Wenzhou Medical University, Wenzhou City, China. Of these, 69 patients $(0.023 \%)$ had PS and $33.33 \%$ of the patients with PS also presented with retinal detachment; therefore, a total of 23 patients ( 23 eyes) comprised the study population (Table 1 ).

\section{Clinical Characteristics}

The main characteristics of the study participants are presented in Table 1. This study included 13 female and 10 male patients from 19 to 57 years old (mean age $29.5 \pm 9.24$ years), $78 \%$ of which were between 21 and 40 years old. Unilateral involvement was present in all of the patients. Ocular pain $(78 \%)$ and blurred vision $(87 \%)$ were the two most commonly reported symptoms. Interestingly, there were two patients (9\%) without symptoms whose condition was identified just through routine physical examination. Around $26 \%$ patients were diagnosed during their first visit, while the remaining $74 \%$ patients were initially diagnosed with and treated for other retinal diseases (e.g. CSC with 7 cases, neuropapillitis with 10 cases ). $69.5 \%$ of the patients visited the clinic within one month of symptom onset. At the time of presentation, four patients (17\%) were documented as having systemic associations including antinuclear antibody positivity (ANA+), hypertension, allergic rhinitis, and Sjogren's syndrome. 18 patients (78\%) had a single episode of PS and five patients had multiple episodes of PS, less than 4 episodes .

\section{Vision Decreases}

The BCVA, logMAR was determined for all patients. As described in Table 2, two patients (9\%) had a BCVA of 0 upon presentation, nine patients $(39 \%)$ were below 0.5 , five patients $(22 \%)$ between $0.5-1$, and seven patients $(30 \%)$ over 1 . No patients were found to be blind or light perception using this test. The mean of the LogMAR visual acuity was $0.61 \pm 0.54$ in patients with PS. In their other eye, 20 patients ( $87 \%$ ) had a BCVA of 0 and three patients (13\%) were between $0.08-0.2$ ( $0.017 \pm 0.045$ average). As expected, the BCVA in PS-affected eyes was worse than in the unaffected eyes. The VA decrease in PS was not related to the unaffected eye according to Pearson's correlation coefficient $(r=0.105 ; p=0.635)$.

\section{Anterior Segment}

PS occurred in 12 patients (52\%) with associated anterior scleritis, which can cause symptoms of conjunctival congestion and tenderness in the superficial sclera (Fig. 2A). UBM examination confirmed the presence of swelling and hyperemia in the superficial fasciitis and sclera at the 12 o'clock (Fig. 2B). Anterior uveitis occurred in eight patients (24\%) with PS as indicated by anterior chamber cell or flare at the time of presentation. There were no hypopyon, synechia, or cataracts found in any of the cases. In the unaffected eye, the anterior ocular portion remained within expected limits. Ocular hypertension was detected neither in the PS-affected eye $(14.21 \pm 6.61 \mathrm{mmHg}$ average $)$ nor in the unaffected one $(15.10 \pm 2.36 \mathrm{mmHg})$. No statistically significant differences were observed in IOP between the PS-affected and unaffected eyes.

\section{Posterior Segment}


A number of abnormalities were observed in PS-affected eyes. All of the patients displayed annular macular retinal detachment (Fig. 1A, 2C, white arrows) but varied in area and sizes (e.g. Case 14 was larger than that Case 1). All patients were tested using OCT scanning, which confirmed serous detachment over the macula (Fig. 1C, 2E) and no abnormal findings were detected in the unaffected eye (Fig. 1G). In order to figure out whether retinal detachment was due to exudation because of retinal pigment epithelium cell (RPE) dysfunction, FFA examination was performed and no fluorescein leakage was observed around the macula regardless of stage (Fig. 1B, 2D). The only positive association identified was between mild fluorescein leakage around the optic nerve and optic disc edema (Fig. 1A). Mild optic nerve swelling was observed in 10 patients ( $43 \%$ ) and about 15 patients $(65 \%)$ were found to have retinal phlebectasia from the fundus examination (Fig. 1A). No choroidal detachment or choroidal folds were observed in this study. In the unaffected eye, fundus imaging revealed typical normal structures (Fig. 1E), which were further confirmed by FFA and OCT examination (Fig. 1F, 1G).

\section{Ultrasonography Examination}

B-scan ultrasonography is the most useful confirmatory analysis for PS diagnosis[12], so all eyes had been examined with B-scan ultrasound. The longitudinal B-mode ultrasound scan showed diffuse posterior coats thickening (PCT) with fluid both in the Tenon's capsule and around the nerve sheath, which displaced as annular hypoechoic in the posterior (Fig. 1D, 2F, black arrows). So, a typical T-sign was observed in all cases (Fig. 2G, white hollow shape). Furthermore, a B-scan ultrasound confirmed the retinal detachment (Fig. 2F, white triangle) and optic nerve swelling in 10 cases (Fig. 2G). In the unaffected eyes, the B-scan ultrasound revealed no abnormality (Fig. $1 \mathrm{H}$ ). The average of the PCT was $2.51 \pm 0.81 \mathrm{~mm}$ in the PS-affected eye, compared to $1.09 \pm 0.29 \mathrm{~mm}$ in the unaffected eye; this difference reached statistical significance $(\mathrm{p}<$ 0.0001 ) (Table 3). From AL measurement, we found that the AL in the PS-affected eye was $22.94 \pm 1.24$ $\mathrm{mm}$ and about $24.4 \pm 0.58 \mathrm{~mm}$ in the unaffected eye on average (Table 3). The increase in PCT was related to the change in $A L$, determined through Pearson's correlation coefficient $(R=-0.795 ; p<0.001)$ but not related to the BCVA $(R=0.117, p=0.596)$.

\section{Choroidal Thickness Changes in the Macula}

The CT of the subfoveal area was examined by EDI-OCT (Fig 1C, 1G, black arrows). In the macular region, the mean CT was highest at the subfoveal level in both eyes and it decreased away from the fovea. The average thickness of the subfoveal choroid was $442.61 \pm 55.61 \mu \mathrm{m}$ in the PS-affected eye and $246 \pm$ $42.31 \mu \mathrm{m}$ in the unaffected eye respectively; this difference had statistical significance $(t=31.8, p<0.0001)$ (Table 3). In order to explore the cause of the increased CT in PS, we analyzed correlations between changes in CT and changes in BCVA, IOP, PCT, and AL. At the subfoveal level, there were significant relationships between CT and PCT according to Pearson's correlation coefficient (Fig. 2H, p = 0.783, $p<$ $0.001)$ and also between $C T$ and $A L(p=-0.65, p=0.001)$; while there were no significant associations between CT and BCVA $(R=0.181, p=0.409)$ and also no correlation with IOP $(R=0.59, p=0.79)$.

\section{Discussion}


This study analyzed the clinical features of PS with serous retinal detachment in 23 Han Chinese patients. Demographically, participants in our study were much younger at disease onset ( 29.5 years, $78 \%$ below 40 years old) compared to a previous study performed in England[12] (49.3 years) but similar to a report from Yang et al. (29.2years) also based on Chinese patients [2]. This finding suggests that young adults might have a higher risk of developing PS in the Chinese population.

It had been reported that systemic or local diseases may contribute to the development of PS [2]. The present study found that $17 \%$ of patients with PS had systemic or local diseases, which was lower than described in studies conducted in England[12] and Singapore (37\%)[4] but, again, consistent with the study in China (13\%)[2]. These results suggest that the association between PS and autoimmune diseases may be weaker in China than in England and Singapore.

In the present study, we found that while blurred vision was one of the most common symptoms of PS, but the BCVA (logMAR) remained below 0.5 in half of our patients. McCluskey et al. (year) also found that around $17 \%$ of patients were not affected visually and that those older than 50 years old had a higher risk of experiencing vision loss [13]. Similarly, Yang et al. (year) reported that around $61.4 \%$ of PS patients were observed with decreased vision. Taken together, these results indicate that BCVA might not be severely affected in PS, especially in its early stages. Potential explanations are: the relatively young age of the patients in our study; the original inflammation occurring in the sclera, not in the retina; and, the short duration between onset and diagnosis reported by the patients in this study.

Many studies have indicated that PS is highly associated with anterior scleritis at rates that range from $20 \%$ to $80 \%$ overall[ $[3,4,12]$; specifically, $19 \%$ in Singapore[4], $59 \%$ in England, $24 \%$ in China[2] and $52 \%$ in this current study. The incidence of comorbidity between PS and anterior scleritis seems to be higher in this report than previously documented. However, Yang et al. and Wieringa et al. used the term "panscleritis" to describe the scleritis that involves both the anterior and posterior segments $[2,14]$. This difference in the definition of PS might contribute to the inconsistencies observed between these studies. Taken together, these results beg the question of whether the inflammation of the anterior scleritis is secondary to the posterior segments and which risk factors are involved.

As expected, physical manifestations of PS varied in this study and included swollen discs, retinal neuroepithelial folds, macular edema, serous retinal detachment, and more. Importantly, we found $74 \%$ patients were initially misdiagnosed. This observation hints at the importance of differential diagnosis for PS with retinal detachment. In this and previous studies[12, 15], B-scan documented a range of ocular abnormalities in patients with PS corresponding to the fundus examination, thereby, ultrasonography is a confirming examination tool potentially necessary for the accurate diagnosis of PS with the typical T sign and posterior coats thicken. However, posterior coats thickening can occur under other conditions (e.g. presence of a tumor[16]), so we suggest that PS should be diagnosed using well-established symptoms and B-scan results.

Recently, an abnormal increase in CT has been hypothesized to contribute to CSC[17], primary open angle glaucoma (POAG)[18], and primary angle closure glaucoma (PACG)[13]. Given its anatomical location, 
scleral inflammation can spread to the choroid, so CT was supposed to be affected due to inflammation in PS. In a previous study, Hiroaki et al reported that the subfoveal CT had grown to $418 \mu \mathrm{m}$ in PS-affected eyes by the initial examination[11], which is in line with our work (average of $442.61 \mu \mathrm{m}$ ). These observations further support that the increased CT might result from inflammation in PS but future studies that focus on the role of the immune system are needed to draw causal connections. Research on other diseases has revealed associations between pathological CT increases and clinical factors, such as AL, choroidal inflammation, and IOP[19]. Based on data from this study, CT correlated with AL and PCT but not with IOP and BCVA. Given that the CT measurement included portions of the eyeball, the correlation between CT and PCT is intuitive. Since PCT was expected to positive correlate with inflammation in PS[12], CT likely represents an additional and promising predictive factor. Some researchers have reported CT increases during the active inflammation period that decreased with anti-inflammation treatment over time[10, 11]. Despite the promising nature of the CT results for monitoring inflammation in PS, it does not seem to be related to BCVA. However, due to the limitations imposed by this study's sample size, further efforts are needed to elucidate the role of CT in PS pathology.

In addition to small study size, this study has some limitations. The retrospective nature of this study greatly limits the significance. And we rule out the infectious factor which was supposed to be associated with PS. Furthermore[20], most patients were referred by other hospitals and our study population may, therefore, represent the most serious cases.

\section{Conclusions}

In conclusion, our study characterized the clinical features of posterior scleritis with retinal detachment, which frequently manifested as ocular pain and blurred vision in symptom, while serous retinal detachment and optic nerve swelling in sign. Typical T-sign detected by B-scan ultrasound provided a critical indicator for diagnosis. Pathological increases in CT and PCT might be a potential predictive factor for inflammation.

\section{Declarations}

The authors report no conflicts of interest.

Ethics approval and consent to participate: This study was approved by the Ethical Committee of the Second Affiliated Hospital of Wenzhou Medical University. All written informed consent was obtained from all participants involved in the study.

Consent to publish: All authors read and approved this manuscript to be published. The authors alone are responsible for the content and writing of the article.

Availability of data and materials: The data were presented within the manuscript, All of the data could be shared, and could also contact the correspondence author for details.

Competing Interest: The authors report no conflicts of interest. 
Funding: this article was supported by the fund of natural science foundation of Zhejiang province (No:LY18H120009)

\section{Authors' Contributions:}

D.Z. and G.Y. were involved with the design of the research. D.Z. and Z.H. assembled and analyzed the data. J.L, Z.Y. and D.Z. performed the statistical analyze. D.Z and J.L and Z.H reviewed the data and participated in the writing of the manuscript. All authors read and approved the final manuscript.

Acknowledgements: none

\section{References}

1. Biswas J, Mittal S, Ganesh SK, Shetty NS, Gopal L: Posterior scleritis: clinical profile and imaging characteristics. Indian journal of ophthalmology 1998, 46(4):195-202.

2. Yang P, Ye Z, Tang J, Du L, Zhou Q, Qi J, Liang L, Wu L, Wang C, Xu M et al: Clinical Features and Complications of Scleritis in Chinese Patients. Ocular immunology and inflammation 2016:1-10.

3. Shenoy R, Suryawanshi M, Isaac R, Philip SK: Posterior scleritis in pediatric age group: A case report and review of literature. Oman journal of ophthalmology 2016, 9(1):59-62.

4. Lavric A, Gonzalez-Lopez JJ, Majumder PD, Bansal N, Biswas J, Pavesio C, Agrawal R: Posterior Scleritis: Analysis of Epidemiology, Clinical Factors, and Risk of Recurrence in a Cohort of 114 Patients. Ocular immunology and inflammation 2016, 24(1):6-15.

5. Ozkaya A, Alagoz C, Koc A, Ozkaya HM, Yazici AT: A case of nodular posterior scleritis mimicking choroidal mass. Saudi journal of ophthalmology : official journal of the Saudi Ophthalmological Society 2015, 29(2):165-168.

6. Liu AT, Luk FO, Chan CK: A case of giant nodular posterior scleritis mimicking choroidal malignancy. Indian journal of ophthalmology 2015, 63(12):919-921.

7. Rossiter-Thornton M, Rossiter-Thornton L, Ghabrial R, Azar DA: Posterior scleritis mimicking orbital cellulitis. The Medical journal of Australia 2010, 193(5):305-306.

8. Mallick J, Pujahari S, Maharana PK: Posterior scleritis presenting as conjunctivitis in a child. BMJ case reports 2016, 2016.

9. Taki W, Keino H, Watanabe T, Okada AA: Enhanced depth imaging optical coherence tomography of the choroid in recurrent unilateral posterior scleritis. Graefe's archive for clinical and experimental ophthalmology = Albrecht von Graefes Archiv fur klinische und experimentelle Ophthalmologie 2013, 251(3):1003-1004. 
10. Hirukawa K, Keino H, Watanabe T, Okada AA: Enhanced depth imaging optical coherence tomography of the choroid in new-onset acute posterior scleritis. Graefe's archive for clinical and experimental ophthalmology = Albrecht von Graefes Archiv fur klinische und experimentelle Ophthalmologie 2013, 251(9):2273-2275.

11. Uchihori H, Nakai K, Ikuno Y, Gomi F, Hashida N, Jo Y, Nishida K: Choroidal observations in posterior scleritis using high-penetration optical coherence tomography. International ophthalmology 2014, 34(4):937-943.

12. McCluskey PJ, Watson PG, Lightman S, Haybittle J, Restori M, Branley M: Posterior scleritis: clinical features, systemic associations, and outcome in a large series of patients. Ophthalmology 1999, 106(12):2380-2386.

13. Chen S, Wang W, Gao X, Li Z, Huang W, Li X, Zhou M, Zhang X: Changes in choroidal thickness after trabeculectomy in primary angle closure glaucoma. Investigative ophthalmology \& visual science 2014, 55(4):2608-2613.

14. Wieringa WG, Wieringa JE, ten Dam-van Loon NH, Los LI: Visual outcome, treatment results, and prognostic factors in patients with scleritis. Ophthalmology 2013, 120(2):379-386.

15. Cheung CM, Chee SP: Posterior scleritis in children: clinical features and treatment. Ophthalmology 2012, 119(1):59-65.

16. Singh AD, Kaiser PK, Sears JE: Choroidal hemangioma. Ophthalmology clinics of North America 2005, 18(1):151-161, ix.

17. Matet A, Daruich A, Zola M, Behar-Cohen F: RISK FACTORS FOR RECURRENCES OF CENTRAL SEROUS CHORIORETINOPATHY. Retina (Philadelphia, Pa) 2017.

18. Sacconi R, Deotto N, Merz T, Morbio R, Casati S, Marchini G: SD-OCT Choroidal Thickness in Advanced Primary Open-Angle Glaucoma. Journal of glaucoma 2017, 26(6):523-527.

19. Kara N, Baz O, Altan C, Satana B, Kurt T, Demirok A: Changes in choroidal thickness, axial length, and ocular perfusion pressure accompanying successful glaucoma filtration surgery. Eye (London, England) 2013, 27(8):940-945.

20. Miranda AF, Cardoso J, Marques N, Barros S, Telles $\mathrm{P}$, Campos N: Isolated posterior scleritis associated with tuberculosis. Arquivos brasileiros de oftalmologia 2016, 79(2):111-112.

\section{Table 1. Symptoms, Clinical Signs At Presentation And Associated Systemic Condition For Each Of The 23 Patients Presenting With Serous Retinal Detachment In Macular}




\begin{tabular}{|c|c|c|c|c|c|c|c|c|c|c|}
\hline NO & Sex & Age & Ocular pain & $\begin{array}{l}\text { Blurred } \\
\text { vision }\end{array}$ & $\begin{array}{l}\text { Anterior } \\
\text { scleritis }\end{array}$ & $\begin{array}{l}\text { AC cell or } \\
\text { flare }\end{array}$ & $\begin{array}{l}\text { Retinal } \\
\text { Phlebectasia }\end{array}$ & $\begin{array}{l}\text { Annular } \\
\text { Retinal } \\
\text { detachment }\end{array}$ & $\begin{array}{l}\text { Optic nerve } \\
\text { swelling }\end{array}$ & $\begin{array}{l}\text { Systemic } \\
\text { association }\end{array}$ \\
\hline 1 & M & $20-30$ & Yes & 1 & Yes & Yes & Yes & Yes & Yes & No \\
\hline 2 & $M$ & $20-30$ & Yes & 0.1 & Yes & Yes & Yes & Yes & Yes & No \\
\hline 3 & $\mathrm{~F}$ & $40-50$ & Yes & 0.5 & Yes & No & Yes & Yes & Yes & ANA+ \\
\hline 4 & $\mathrm{~F}$ & $20-30$ & Yes & 0.2 & Yes & Yes & Yes & Yes & Yes & No \\
\hline 5 & $\mathrm{~F}$ & $18-20$ & Yes & 0.7 & No & Yes & Yes & Yes & Yes & No \\
\hline 6 & $M$ & $20-30$ & Yes & 0.4 & Yes & Yes & Yes & Yes & No & No \\
\hline 7 & $\mathrm{~F}$ & $30-40$ & Yes & 0.7 & No & No & Yes & Yes & No & No \\
\hline 8 & $\mathrm{~F}$ & $50-60$ & Yes & 0 & No & No & No & Yes & No & No \\
\hline 9 & $\mathrm{~F}$ & $20-30$ & Yes & 0.5 & No & No & Yes & Yes & Yes & No \\
\hline 10 & $\mathrm{~F}$ & $20-30$ & Yes & 1.1 & No & No & No & Yes & No & No \\
\hline 11 & $M$ & $20-30$ & Yes & 0 & Yes & Yes & Yes & Yes & No & No \\
\hline 12 & M & $20-30$ & Yes & 0.5 & No & No & No & Yes & No & No \\
\hline 13 & $M$ & $20-30$ & Yes & 1.2 & No & No & No & Yes & Yes & Rhinallergosis+ \\
\hline 14 & $\mathrm{~F}$ & $20-30$ & Yes & 0.2 & Yes & No & No & Yes & No & No \\
\hline 15 & M & $30-40$ & No & 1.7 & Yes & No & Yes & Yes & No & No \\
\hline 16 & $\mathrm{~F}$ & $18-20$ & No & 1 & No & No & Yes & Yes & No & Hypertension+ \\
\hline 17 & $\mathrm{~F}$ & $30-40$ & Yes & 0.4 & No & No & Yes & Yes & No & No \\
\hline 18 & $\mathrm{~F}$ & $40-50$ & No & 2 & No & No & No & Yes & No & SS+ \\
\hline 19 & M & $20-30$ & Yes & 0.1 & No & No & No & Yes & No & No \\
\hline 20 & $\mathrm{~F}$ & $20-30$ & Yes & 0.2 & Yes & Yes & No & Yes & No & No \\
\hline 21 & $\mathrm{~F}$ & $20-30$ & Yes & 1.3 & Yes & Yes & Yes & Yes & Yes & No \\
\hline 22 & M & $30-40$ & No & 0 & Yes & Yes & Yes & Yes & Yes & No \\
\hline 23 & $\mathrm{M}$ & $30-40$ & No & 0.1 & Yes & No & Yes & Yes & Yes & No \\
\hline \multicolumn{3}{|c|}{$\begin{array}{l}\text { Number/percentage } \\
(\mathrm{N} / \%)\end{array}$} & 18/(78.26\%) & $20 /(86.96 \%)$ & $12 /(52.17 \%)$ & 8/(23.78\%) & $15 /(65.21 \%)$ & $23 /(100 \%)$ & $10 /(43.48 \%)$ & $4 /(17.39 \%)$ \\
\hline
\end{tabular}

Table 2. The Basic Clinical Features Of Posterior Scleritis In 23 Cases 


\begin{tabular}{|c|c|c|c|}
\hline \multirow{2}{*}{$\begin{array}{l}\text { Clinical features } \\
\text { Age }\end{array}$} & & eyes & percent]\%ם \\
\hline & 2 & \multicolumn{2}{|r|}{8.69} \\
\hline $\begin{array}{l}\text { Below } 20 \text { years } \\
\text { Between } 21-40 \text { years } \\
\text { Older 40years } \\
\text { Gender }\end{array}$ & $\begin{array}{l}18 \\
3\end{array}$ & \multicolumn{2}{|r|}{$\begin{array}{l}78.26 \\
13.04\end{array}$} \\
\hline Male & \multicolumn{2}{|c|}{10} & 43.47 \\
\hline \multicolumn{2}{|c|}{$\begin{array}{l}\text { Complaint vision loss } \\
\text { Diagnosis rate } \\
\text { Onset time } 1 \text { month } \square\end{array}$} & $\begin{array}{l}3 \\
20 \\
6 \\
16\end{array}$ & $\begin{array}{r}56.53 \\
78.26 \\
86.96 \\
26.09 \\
69.56\end{array}$ \\
\hline \multicolumn{4}{|c|}{ Visual acuity (logMAR) } \\
\hline \multicolumn{2}{|c|}{ Less than 0} & 2 & 8.96 \\
\hline \multicolumn{2}{|l|}{ Below 0.5} & 9 & 39.13 \\
\hline \multicolumn{2}{|l|}{ Between 0.5 and 1} & 5 & 21.73 \\
\hline \multicolumn{2}{|l|}{ Over 1} & 7 & 30.43 \\
\hline \multicolumn{2}{|c|}{$\frac{\text { Light perception or blind }}{\text { Total }}$} & $\frac{0}{23}$ & $\frac{0}{100}$ \\
\hline
\end{tabular}

\section{Table 3. Clinical Measurement In Posterior Scleritis And Fellow Eye $(\mathrm{N}=23)$}

\begin{tabular}{|c|c|c|c|c|c|c|c|c|}
\hline \multirow[b]{2}{*}{ Isurement } & \multicolumn{2}{|c|}{ Posterior Scleritis } & \multicolumn{2}{|c|}{ Fellow eye } & \multicolumn{4}{|c|}{ Difference } \\
\hline & Mean & SD & Mean & SD & Mean & $95 \% \mathrm{Cl}$ & $\mathrm{t}$ & $P^{*}$ \\
\hline visual acuity & 0.61 & 0.54 & 0.017 & 0.045 & 0.593 & 0.36 to 0.83 & 5.03 & 0.000 \\
\hline \multirow[t]{2}{*}{$\mathrm{Hg}$} & 14.21 & 6.61 & 15.1 & 2.36 & -0.89 & -2.5 to -0.69 & -1.19 & 0.248 \\
\hline & 22.94 & 1.24 & 24.4 & 0.58 & -1.46 & -1.98 to -0.92 & -5.64 & 0.000 \\
\hline thickness, $\mu \mathrm{m}$ & 442.61 & 55.61 & 246 & 42.31 & 196.61 & 183.78 to 209.43 & 31.8 & 0.000 \\
\hline thickness $\square \mathrm{mm}$ & 2.57 & 0.81 & 1.09 & 0.29 & 1.48 & 1.26 to 1.93 & 9.8 & 0.000 \\
\hline
\end{tabular}

Data are expressed as means $\pm \mathrm{SD}$. AL, axial length; IOP, intraocular pressure; $\mathrm{Cl}$, confidence interval. *P values in boldface indicate $\mathrm{P}[0.05$, and considered significant.

\section{Figures}

Figure 1

Clinical findings in Case 1. A: Fundus imaging of disc swelling, retinal phlebectasia, and serous retinal detachment (white arrow) at the macula B: Fluorescein leakage around the optic nerve at the late stage in 
fundus fluorescein angiography; C: EDI-OCT scanning of serous detachment over the macula. The short arrows indicate the chorioscleral interface and red double arrowheads indicate subfoveal CT. The CT of the left eye measured $607 \mu \mathrm{m}$ at the initial visit, determined by EDI-OCT. D: Ultrasound scan of scleral thickening, black arrows denote the posterior boundary of the sclera. E, F, G, H: demonstrated normal

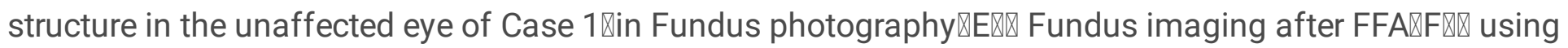
EDI-OCT revealed $461 \mu \mathrm{m}$ choroidal thickness, indicated by short arrows $₫$ G $\llbracket$ and B-scan ultrasonography

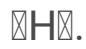

\section{Figure 2}

Clinical findings of poster scleritis with serous retinal detachment. A: Anterior segment examination showed conjunctival congestion and anterior scleritis with tenderness in the upper part of the sclera. B: UBM examination detected swelling and hyperemia in the superficial fasciitis and sclera at the 12 o'clock. C: Posterior segment examination revealed retinal detachment at the macula, indicated by white arrow. D: FFA illuminated the serous retinal detachment at the macula but no fluorescein leakage was detected after about fifty minutes E: The OCT scanning confirmed serous retinal detachment at the macula. F: B-scan ultrasonography confirmed scleral thickening, black arrows indicate the posterior boundary of the sclera and the retinal detachment is indicated by the white triangle G: B-scan ultrasonographyshowing diffuse thickening of the posterior coats of the eye $(3.6 \mathrm{~mm})$ together with fluid in the Tenon's capsule (white arrow). The "T" sign is indicated by the white hollow shape. H: The increases in the PCT correlated positively with the increasing $C T(p=0.783, p<0.001)$. 